\title{
OPEN Low cost, high performance ultrafiltration membranes from glass fiber-PTFE-graphene composites
}

\author{
Umar Khan ${ }^{1}$, Sonia Biccai ${ }^{2}$, Conor S. Boland ${ }^{2} \&$ Jonathan N. Coleman ${ }^{2 凶}$
}

The development of low-cost ultrafiltration membranes with relatively high flow rate and selectivity is an important goal which could improve access to clean water in the developing world. Here we demonstrate a method to infuse mixtures of graphene nanosheets and Teflon nanoparticles into ultracheap glass fibre membranes. Annealing the resultant composites leads to coalescence of the Teflon, resulting in very stable membranes with significantly enhanced mechanical properties. In filtration tests, while adding 10 wt\% graphene/Teflon to the glass fibre membrane decreased the flow rate by $\times 100$, the selectivity improved by $\times 10^{3}$ compared to the neat glass fibre membrane. This combination of selectively and flow rate was significantly better than any commercial membrane tested under similar circumstances. We found these membranes could remove $>99.99 \%$ of $25-250 \mathrm{~nm}$ diameter SiC nanoparticles dispersed in ethanol, transmitting only particles with diameters $<40 \mathrm{~nm}$, performance which is superior to commercial alumina membranes. Field trials on dirty canal water showed these composite membranes to remove aluminium to a level $\times 10$ below the EU limit for drinking water and reduce iron and bacteria contents to below detectable levels.

Access to clean water is one of the greatest problems currently facing humanity. Globally, approximately two billion people use contaminated drinking water daily ${ }^{1}$ while another 750 million people do not have reliable access to drinking water ${ }^{2}$. As a result, over three million people die annually due to water-related illnesses ${ }^{3}$. While access to clean drinking water is not usually a problem in the developed world, demands for water are growing rapidly. It is estimated that industry is responsible for $\sim 40 \%$ of demand for water in the $\mathrm{EU}^{4}$. The global demand of industrial water is expected to increase by $400 \%$ by $2050^{2}$. Clearly, solutions must be found to meet the demand for both drinking and industrial water. One important solution to both these problems is to use filtration to purify both dirty and waste water . $^{5}$

Water purification by filtration typically uses porous, permeable membranes to separate dispersed solids such as particles, macromolecules and micro-organisms from the water. In general, the water to be treated is passed through the membrane resulting in solids above a certain cut-off size remaining in the retentate while the purified water (containing only solids smaller than the cut-off size) passing through the membrane. The cutoff size depends on the pore size distribution within the membrane. In all cases, membranes need to maximise both selectivity (i.e. the ability to remove as much impurity material as possible) and flow rate (the rate at which purified water can be produced). In addition, membranes material must be mechanically robust, chemically stable and cost effective.

A number of different membranes types are widely used. Fibrous membranes, are generally polymeric in nature and are commonly made using electrospinning ${ }^{6-8}$. These membranes often suffer from bio and chemical fouling both on the surface and deep inside the structure ${ }^{9,10}$, and are limited by the mechanical, thermal and chemical instabilities typical of polymers ${ }^{11}$. Similar to fibrous membranes are foam structures which are also polymeric and often fabricated from cellulose acetate or nylon. As a result, these membranes tend to suffer from the same problem/solutions as fibrous membranes. Ceramic membranes tend to be fabricated from alumina or other metal oxides and are made using sol-gel techniques ${ }^{12}$. They have very good thermal stability but are brittle, have low acid/ base stability ${ }^{13}$ and can be very expensive.

More recently, attention has moved to nano-material-based membranes, often incorporating $1 \mathrm{D}^{14,15}$, or more relevant here, $2 \mathrm{D}$ materials such as graphene and graphene oxide $(\mathrm{GO})^{16-22}$ or even $2 \mathrm{D}$ polymers ${ }^{23}$, for use in 

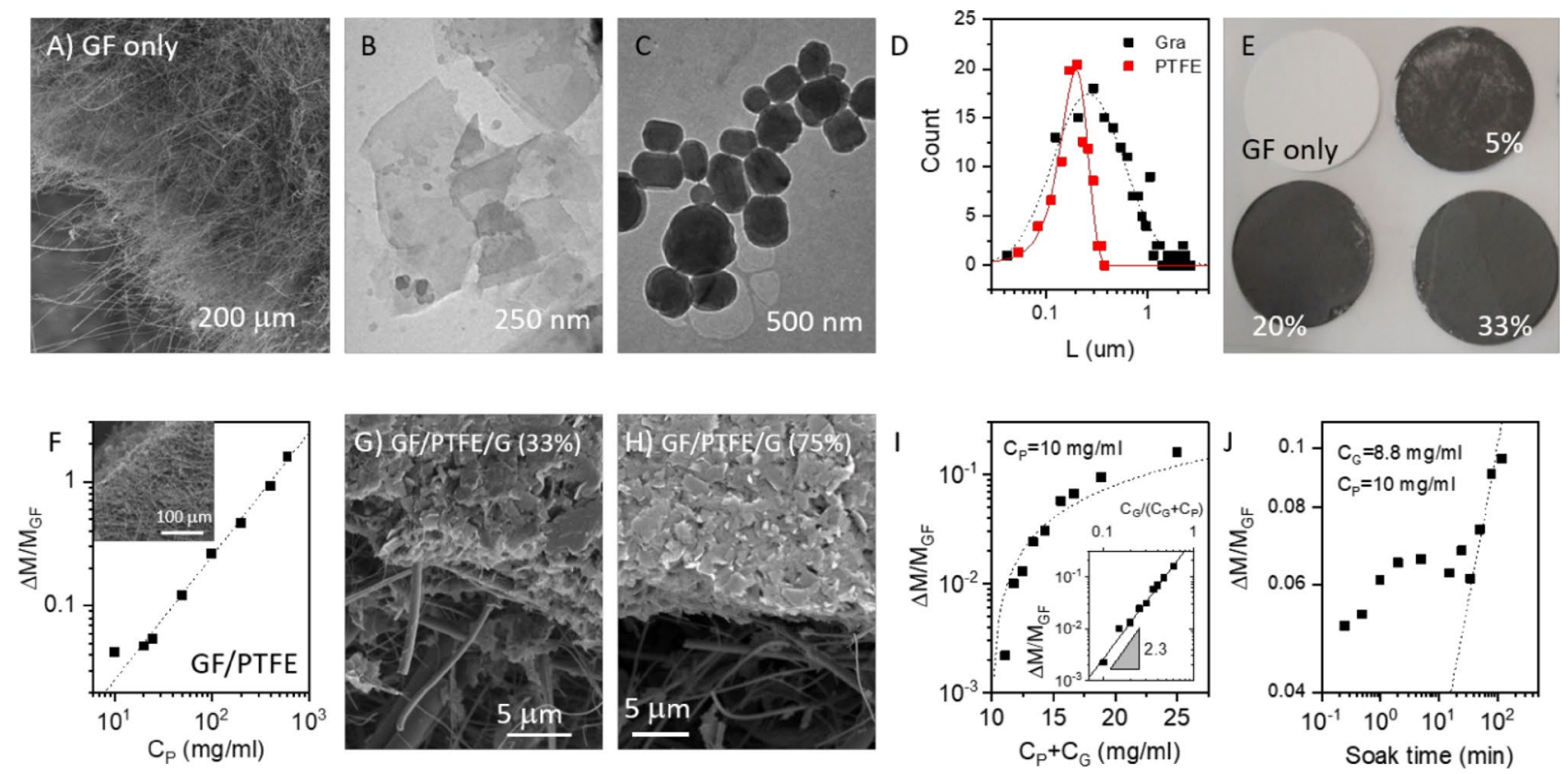

Figure 1. (A) SEM image of the edge of a glass fibre (GF) membrane. (B,C) TEM images of typical LPE graphene nanosheets (B) and PTFE nanoparticles (C). (D) Lateral size distribution for both graphene nanosheets and PTFE nanoparticles. (E) Photographs of GF membrane (top left) and membranes with a range of nominal PTFE/graphene contents. The number reflects the graphene content in the soak solution expressed as $\mathrm{C}_{\mathrm{G}} /\left(\mathrm{C}_{\mathrm{G}}+\mathrm{C}_{\mathrm{P}}\right)$ where $\mathrm{C}_{\mathrm{G}}$ and $\mathrm{C}_{\mathrm{P}}$ are the concentrations of graphene and PTFE in the soak solutions. $(\mathrm{F})$ The membranes are soaked in a dispersion of PTFE nanoparticles with the mass uptake plotted versus the concentration of PTFE (soak time $45 \mathrm{~min}$ ). The dashed line represents linearity. Inset: SEM images of a GF membrane partially filled with PTFE. (G,H) GF membrane partially filled with PTFE and graphene where $\mathrm{C}_{\mathrm{G}} /$ $\left(\mathrm{C}_{\mathrm{G}}+\mathrm{C}_{\mathrm{P}}\right)=33 \%(\mathbf{G})$ and $74 \%(\mathbf{H}) .(\mathbf{I}, \mathbf{J})$ Graphs showing fractional mass uptake on soaking for two soaking scenarios: (I) The membranes are soaked in a dispersion of both PTFE nanoparticles and graphene nanosheets with $C_{P}=10 \mathrm{mg} / \mathrm{ml}$ and varying $C_{G}$. The mass uptake is plotted versus the combined concentration of PTFE and graphene (soak time $45 \mathrm{~min}$ ). The dashed line represents a linear increase in mass uptake with $\mathrm{C}_{\mathrm{G}}$. Inset: Mass uptake poltted as a function of $\mathrm{C}_{\mathrm{G}} /\left(\mathrm{C}_{\mathrm{G}}+\mathrm{C}_{\mathrm{P}}\right)$ showing a clear power law dependence. $(\mathrm{J}) \mathrm{The}$ membranes are soaked in a dispersion of both PTFE nanoparticles and graphene nanosheets with $\mathrm{C}_{\mathrm{P}}=10 \mathrm{mg} / \mathrm{ml}, \mathrm{C}_{\mathrm{G}}=8.8 \mathrm{mg} /$ $\mathrm{ml}$ for varying soak times. The dashed line represents what would be expected if the uptake was solely diffusion limited.

filtration. For example, a large number of papers have described GO membranes with very high selectivity for water purification ${ }^{24-26}$. In addition, a number of researchers have mixed graphene with polymer matrices, yielding highly selective filter membranes with enhanced mechanical properties ${ }^{27,28}$. Alternatively graphene oxide has been combined with inorganic nanoparticles, for example $\mathrm{TiO}_{2}$, in order to control the pore size ${ }^{16}$. Research in 2D materials for filtration has largely focused in high-performance applications such as desalination. However, although these membranes often show significant potential in such high-performance applications, more work is definitely needed on nano-enabled filters for relatively low-end applications such as water purification in the third world. In such fields, extremely high selectivity is perhaps less important than achieving scalability and low cost.

Thus, it is clear that there is an opening for a new low-cost, chemically and mechanically stable membrane material which can combine good flow rate and selectivity. When thinking of high-performance membranes, one does not usually think of glass fibre membranes. These are very low-cost membranes that consist of a non-woven network of glass fibres. While they display very high flow rates, very good thermal and chemical stability, they have poor mechanical properties and very low selectivity. Typically, they are generally used only as pre-filter ${ }^{29,30}$. However, we hypothesised that these low-cost membranes might be combined with a graphene-based nanocomposite to develop membranes with reasonably high performance but low cost. Here we demonstrate a method to modify commercially available glass fibre membrane with solution processable composites of graphene and Teflon to prepare ultra-filtration membranes with tuneable properties. We find combinations of flow rates and selectivity superior to a range of commercial membranes.

\section{Results and discussion}

Composite membrane production and basic characterisation. Commercial glass fibre (GF) membranes are formed from mats of glass fibres, each fibre having diameter of a few microns and a length of many hundreds of microns. They have porosities of $>95 \%$ (i.e. densities of $\sim 200 \mathrm{~kg} / \mathrm{m}^{3}$ ), typical pore sizes of tens of micron and are very cheap to make (see Fig. 1A). However, because of the large pore sizes, although such filters do show high flow rates, they only block large particles. Here we aim to reduce the particle size transmitted through the membrane to the filtrate by using exfoliated graphene nanosheets to reduce the effective pore size. 
We reasoned that soaking the GF membranes in a dispersion of graphene nanosheets would lead to the infusion of nanosheets into the interior of the membrane resulting in the formation of a nanosheet network with small pore size. Importantly, such a network would be mechanically supported by the glass fibres.

To achieve this, we prepared few-layer graphene nanosheets (Fig. 1B) by liquid phase exfoliation ${ }^{31,32}$, a wellknown graphene production technique (see "Methods"). While initial trials showed that graphene could be inserted by soaking the GF membrane in an aqueous, surfactant stabilised dispersion of graphene nanosheets, further experiments showed the graphene network to be very unstable, rapidly flaking of the membrane, especially during filtration trials. Early attempts to introduce a polymer binder by adding a soluble polymer to the graphene dispersion failed to give stable, robust membranes.

To address this problem, we used a novel solution to insert a polymeric binder into the GF membrane, either with or without the presence of graphene. Instead of using a soluble polymer as a binder, we used commercially available Teflon (PTFE) nanoparticles (PTFE-NPs). These nanoparticles are pseudo-spherical (Fig. 1C) and are supplied as an aqueous dispersion. TEM measurements showed both PTFE particles and graphene nanosheets to display a broad distribution of sizes typically in the range of hundreds of nm (Fig. 1D). The mean graphene nanosheet length was $625 \mathrm{~nm}$ while the mean PTFE nanoparticle diameter was $200 \mathrm{~nm}$. To insert the graphene nanosheets and polymer binder into the GF membrane, we placed membranes into aqueous dispersions of either PTFE-NPs or mixtures of PTFE-NPs and graphene nanosheets and allowed them to soak in the liquid for various times. Under these circumstances, the liquid wicks into the porous interior of the GF membranes, bringing the dispersed material (i.e. PTFE-NPs/nanosheets) with it. Such wicking behaviour is essentially an example of capillary action ${ }^{33}$ and is driven by the favourable intermolecular interactions between the liquid and glass fibres. Over time both PTFE-NPs and graphene nanosheets become deposited onto the glass fibres, both at the surface and within the interior of the membrane, resulting in a significant mass uptake. Similar methods have been used to infuse polymer into carbon nanotube-based papers ${ }^{34-36}$. Such deposition was visible to the eye, with any membrane surface which was in contact with the graphene-containing dispersion changing from pure white to black (Fig. 1E). Sintering the resultant composite membranes at $420^{\circ} \mathrm{C}$ resulted in very stable composites due to coalescence of the PTFE-NPs.

It was possible to control the mass uptake $(\Delta \mathrm{M})$ via either the concentration of the soaking dispersion or the soak time. Shown in Fig. $1 \mathrm{~F}$ is data for the fractional mass uptake $\left(\Delta \mathrm{M} / \mathrm{M}_{\mathrm{GF}}\right.$ where $\mathrm{M}_{\mathrm{GF}}$ is the initial mass of the GF membrane) of a GF membrane soaked in PTFE-NP-only dispersions of different concentrations, $\mathrm{C}_{\mathrm{P}}$ (soak time $45 \mathrm{~min}$ ). This data shows a clear linear increase of mass uptake with PTFE concentration. SEM imaging (inset) appeared to show PTFE inside the GF membrane after sintering, with the membranes displaying densities of $\sim 210-850 \mathrm{~kg} / \mathrm{m}^{3}$ consistent with significant porosity retention.

More importantly, we investigated the effect of soaking GF membranes in mixtures of graphene and PTFENPs with individual concentrations of $C_{G}$ and $C_{P}$ and so a combined concentration of $C_{G}+C_{P}$. SEM images of composite membranes formed by soaking GF membranes in mixtures of graphene and PTFE are shown in Fig. $1 \mathrm{G}, \mathrm{H}$. These images show that, although some graphene/PTFE has entered the membrane, the majority has formed a thick layer on top. This shows that under these preparation conditions, the spatial distribution of infused material is non-uniform, with the local concentration of graphene/PTFE-NPs increasing from centre to surface. This observation is almost certainly due to deposition of graphene/PTFE-NPs onto the glass fibres which occurs preferential near the surface of the membrane resulting in the observed gradient of deposited graphene/PTFE-NPs.

We found it impossible to measure the individual masses of graphene and PTFE which had been taken up by the membrane. For example, thermogravimetric analysis failed because we were unable to completely burn off the graphene, possibly because of the poor thermal conductivity of the glass or the strong adhesion of the graphene. In addition, spectroscopic surface techniques such as XPS and Raman proved unsuitable as we cannot be sure that the graphene/Teflon ratio is the same at the membrane surface as in the bulk. This made it impossible to determine the mass of the individual components. As a result, we concentrate on the total mass uptake which could be found by weighing.

In Fig. 1I we plot the factional mass uptake (after sintering) as a function of combined PTFE and graphene concentrations. Here the PTFE concentration was fixed at $C_{P}=10 \mathrm{mg} / \mathrm{ml}$ while the graphene concentration, $C_{G}$, was varied. Interestingly, for reasons we do not understand, the total mass uptake scaled roughly linearly with $\mathrm{C}_{\mathrm{G}}$ (dashed line) suggesting the mass uptake to be dominated by graphene. However, it is clear that some PTFE does enter the membrane and act as a binder as such composite membranes are stable after sintering whereas those membranes prepared without PTFE (graphene only) were not. In the inset of Fig. 1I, we plot the mass uptake versus the mass fraction of graphene in the soak dispersion $\mathrm{C}_{\mathrm{G}} /\left(\mathrm{C}_{\mathrm{G}}+\mathrm{C}_{\mathrm{P}}\right)$, finding a well-defined power law with exponent 2.3. This implies that graphene uptake is favoured over that of polymer and suggests the higher $\Delta \mathrm{M} /$ $\mathrm{M}_{\mathrm{GF}}$ dispersion may have a higher relative graphene constant than those membranes with lower mass uptake.

The fractional mass uptake is shown as a function of soak time in Fig. 1J for a fixed combination of graphene and PTFE soaking concentrations. Interestingly, over the first $10 \mathrm{~min}$ of soaking the mass uptake increases slowly before accelerating and increasing thereafter as $\Delta M / M_{G F} \propto \sqrt{t}$, in line with Washburn's equation ${ }^{33}$, and consistent with diffusion limitations.

Mechanical properties of membranes. For water purification applications, it is important that membranes are mechanically robust and not prone to cracking or other damage. It is possible that the addition of PTFE and graphene can actually improve the mechanical properties of the GF membranes. In particular, graphene is well known to improve the mechanical properties of composites under certain circumstances ${ }^{37}$. To test this, we performed tensile mechanical tests on membranes with a range of different values of $\Delta \mathrm{M} / \mathrm{M}_{\mathrm{GF}}$. In addition, for comparison purposes, we performed the same tests on a number of commercial membranes including 

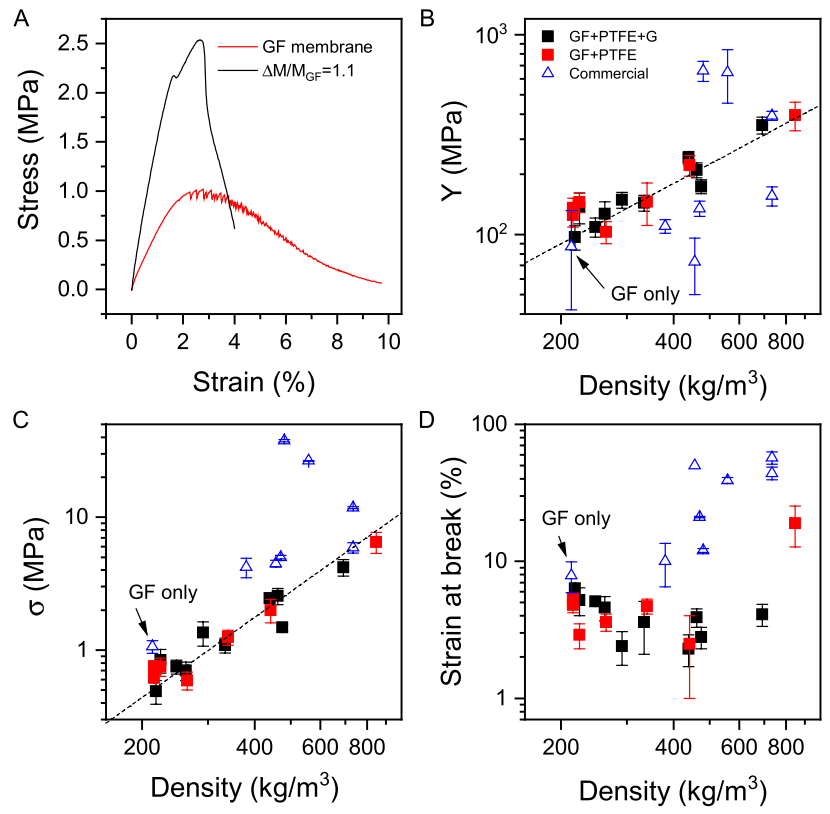

Figure 2. Mechanical properties of membranes. (A) Typical stress-strain curves for a GF membrane and a composite membrane with fractional mass uptake of 1.1. (B-D) Modulus (B), tensile strength (C) and strain at break (D), all plotted versus measure membrane density. Data is plotted for membranes filled with both PTFE and PTFE + graphene. Also shown are data for commercial membranes. The dashed lines in (B,C) represent linear and quadratic behaviour respectively.

a GF-only membrane. Shown in Fig. 2A are representative stress-strain curves for a GF membrane (untreated) and a GF membrane soaked in graphene and PTFE-NPs with $\Delta \mathrm{M} / \mathrm{M}_{\mathrm{GF}}=1.1$. It is clear from these curves that addition of PTFE and graphene improves the mechanical properties of the membranes.

To show this in more detail we extracted three parameters from the stress-strain curves, the modulus, $Y$, the tensile strength, $\sigma$, and the strain at break. We also measured the same parameters for membranes produced by infiltrating PTFE-NPs only into the GF membranes as well as a range of commercial membranes fabricated from teflon, polypropylene, polyester, nylon and cellulose. In order to facilitate comparison, we plot each mechanical property versus the measured density of the membrane under test, keeping in mind that for the composite membranes produced in this study, the density increases linearly with the mass uptake. Shown in Fig. 2B,C are the modulus, strength and strain at break plotted versus membrane density for all tested membranes. Both modulus and strength increase with membrane density and so with $\Delta \mathrm{M} / \mathrm{M}_{\mathrm{GF}}$. In both cases, the data for GF membranes infiltrated with both PTFE-NPs alone and PTFE-NPs -graphene sit on the same trend line indicating the graphene to have little impact on the membrane mechanical properties (probably because most of the graphene is concentrated near the surface of GF membranes). Interestingly, both stiffness and strength follow different power laws with density consistent with $Y \propto \rho$ and $\sigma \propto \rho^{2}$ (dashed lines). While the strength-density scaling is in line with a number of papers on graphene-based aerogel or porous monoliths ${ }^{38,39}$, the modulus-density scaling is inconsistent with most published results which tend to show $Y \propto \rho^{239-41}$. However, the structure of these composite membranes is quite complex with a spatially non-uniform distribution of infused particles so it is probably not surprising that the mechanics are atypical. While modulus and strength improve with infusion, the strain at break decreases slightly as shown in Fig. 2D, falling from $\sim 8 \%$ in GF-only membranes to $~ 3 \%$ in highly-filled membranes. While this indicates an increase in brittleness, this is somewhat compensated by the increase in strength. We note that most of the commercial membranes showed combinations of modulus, strength and stiffness which were somewhat superior to our composite membranes. However, we note that these composite membranes are robust enough for practical use in filtration applications (see below) and are generally superior to GF-only membranes.

Characterising GF/graphene/PTFE-NP composites as ultrafiltration membranes. We have tested composites of GF membranes infiltrated with both PTFE-NPs only as well as mixtures of PTFE-NPs and graphene as ultrafiltration membranes. We first performed experiments to determine whether any graphene leached out of the membranes during filtration. Extensive spectroscopic and microscopic analysis of filtrate showed no evidence of graphene, showing the nanosheets to be well bound to the membranes, probably due to the presence of the PTFE-NPs.

Ultrafiltration testing was performed via filtration experiments using dispersions of $\mathrm{SiC}$ nanoparticles in ethanol $(0.2 \mathrm{mg} / \mathrm{ml})$ as a feed dispersion. We chose SiC nanoparticles over more well-defined nano-particles because their broad size distribution ( $~ 50-200 \mathrm{~nm}$ in diameter) allows us to estimate the size distribution of particles transmitted through the membrane to the filtrate (see below). We filtered the SiC/ethanol dispersions through 

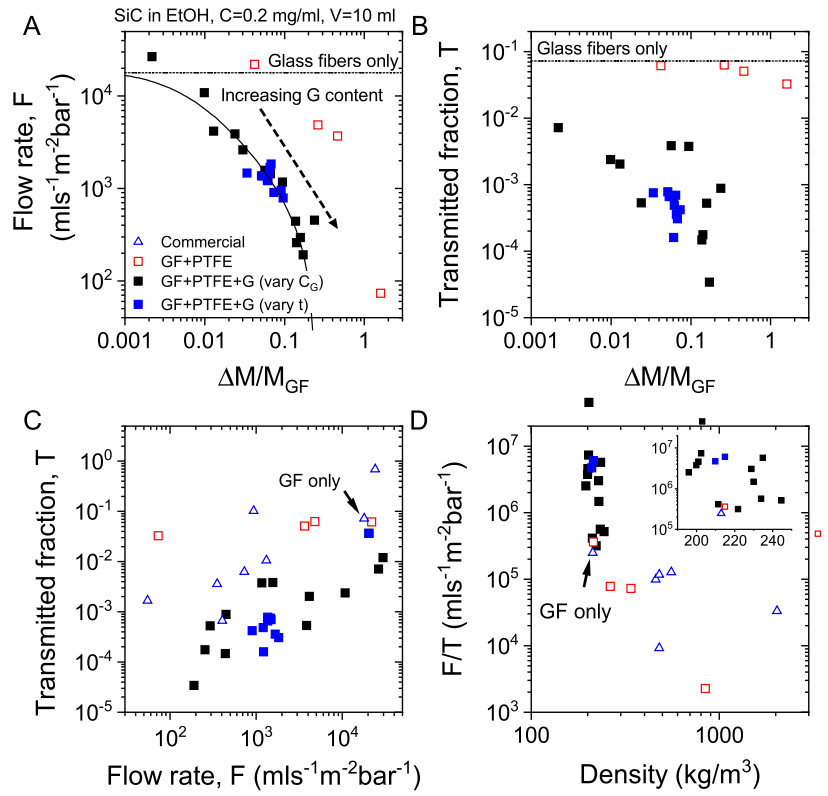

Figure 3. Filtration properties of nanocomposite membranes as tested by filtering an ethanol-based dispersion $(10 \mathrm{ml})$ of $\mathrm{SiC}$ nanoparticles $(0.2 \mathrm{mg} / \mathrm{ml})$ through the membrane $\left(\mathrm{A}=2.23 \mathrm{~cm}^{2}\right)$ mounted on a conventional Buchner funnel $(\mathrm{P}=60 \mathrm{kPa})$. (A,B) Measured flow rate $(\mathbf{A})$ and transmitted fraction $(\mathbf{B})$ plotted as a function of fractional PTFE/Graphene mass uptake for three types of composite membranes (i.e. those in Fig. 1H-J, see legend in panel (C). N.B the transmitted fraction is the fraction of $\mathrm{SiC}$ nanoparticles (by mass) transmitted through the membrane. The solid line in A is a fit to Eq. (4). (C) Transmitted fraction plotted vs. flow rate for a range of composite membranes with the performance of some commercial membranes shown for comparison. N.B in the plot, high performance membranes should be located toward the bottom right. (D) The performance can be approximated by the ratio $\mathrm{F} / \mathrm{T}$, which is plotted versus membrane density. The membranes produced here perform extremely well at low density.

our composite membranes, measuring both the rate of permeate flow, F, (volume per second per unit area per unit pressure drop) through the membrane and the concentration of $\mathrm{SiC}$ in the filtrate (via optical absorbance spectroscopy). Dividing the latter quantity by the concentration of $\mathrm{SiC}$ in the initial dispersion $(0.2 \mathrm{mg} / \mathrm{ml})$ gave the mass fraction of $\mathrm{SiC}$ transmitted through the membrane (T) which is a measure of selectivity.

Shown in Fig. 3A is flow rate data, measured for GF membranes filled with PTFE-NPs-only as well as PTFENPs and graphene at a range of values of $\Delta \mathrm{M} / \mathrm{M}_{\mathrm{GF}}$. The latter type of membranes were prepared in two ways; by varying the concentration of graphene in the soak dispersion as well as varying the soak time. In all cases, as $\Delta \mathrm{M} / \mathrm{M}_{\mathrm{GF}}$ is increased, the flow rate falls off compared to the GF-only commercial membranes. However, the decrease in flow rate was considerably more rapid in the PTFE-NP/graphene membrane compared to those with PTFE-NPs alone. This suggests graphene to significantly influence the pore structure within the membrane.

We can model this behaviour quantitatively via Darcy's law which describes fluid flow through porous media. In its simplest form, this gives an equation for the flow rate $\left[\mathrm{m}^{3} / \mathrm{s}\right], \mathrm{Q}^{42}$ :

$$
Q=\frac{k A \Delta P}{\eta t} \text {. }
$$

where $\mathrm{k}$ is the permeability of the porous medium, $\mathrm{t}$ and $\mathrm{A}$ are its thickness and area, $\Delta \mathrm{P}$ is the pressure drop across the medium and $\eta$ is the fluid viscosity. Because of the way we define $F(F=Q / A \Delta P)$, we can write $F=k / \eta t$. We would expect the permeability to depend on the typical size of the pores as well as the porosity, $\mathrm{P}$. Modelling the pore distribution as fractal, it has been shown that the permeability is given by ${ }^{42}$

$$
k=C A_{P} \frac{P^{m}}{1-P},
$$

where $\mathrm{C}$ is a constant, $\mathrm{A}_{\mathrm{P}}$ is a measure of the cross-sectional area of the pores and $\mathrm{m}$ is the tortuosity factor which lies in the range $1<\mathrm{m}<4$.

In addition, we would expect the porosity to depend on the mass uptake, $\Delta \mathrm{M} / \mathrm{M}_{\mathrm{GF}}$. It is straightforward to show that

$$
P=1-\frac{\rho_{G F-M e m}}{\rho_{G F}}-\frac{\rho_{G F-M e m}}{\rho_{G+P}} \Delta M / M_{G F},
$$

where $\rho_{G F-M e m} / \rho_{G F} \approx 0.026$ is the ratio of the density of the glass fibre membrane to that of the glass fibres alone and $\rho_{G+P}$ is the combined density of the graphene/polymer filler. 
Combining all of these equations yields

$$
F=\frac{C A_{P}}{\eta t} \frac{\left[1-\frac{\rho_{G F-M e m}}{\rho_{G F}}-\frac{\rho_{G F-M e m}}{\rho_{G+P}} \Delta M / M_{G F}\right]^{m}}{\frac{\rho_{G F-M e m}}{\rho_{G F}}+\frac{\rho_{G F-M e m}}{\rho_{G+P}} \Delta M / M_{G F}} .
$$

We have fit this equation to the data in Fig. 3A finding very good agreement once the following fit parameters are used: $A_{P} \sim 6 \times 10^{-14} \mathrm{~m}^{2}, \mathrm{~m}=1, \rho_{G F-M e m} / \rho_{G+P}=4.1$ (assuming $\left.\mathrm{C} \sim 1 / 8 \pi\right)^{42}$. We can estimate the characteristic pore size from $\sqrt{ } A_{P}=250 \mathrm{~nm}$, a value which seems reasonable given the size of the graphene nanosheets. However, we note that this value should be treated with caution as this model cannot account for the inevitable reduction in pore size as $\Delta \mathrm{M} / \mathrm{M}_{\mathrm{GF}}$ is increased.

In addition, the value of $\rho_{G F-M e m} / \rho_{G+P}$ is very high, implying the polymer/graphene network to be even sparser than the GF network. However, this discrepancy is almost certainly due to the spatial non-uniformity of the polymer/graphene due to its concentration at the membrane surface. This fit parameter suggests that, averaged over the whole membrane, $\rho_{\mathrm{G}+\mathrm{P}} \sim 50 \mathrm{~kg} / \mathrm{m}^{3}$. Assuming the true density of the actual polymer/graphene layer is $1000-2000 \mathrm{~kg} / \mathrm{m}^{3}$, this would imply the polymer/graphene layer to occupy $5-10 \%$ of the membrane thickness. From Fig. 1G,H, we can estimate the polymer/graphene layer thickness to be $\sim 5-10 \mu \mathrm{m}$ thick. Because the GF membrane thickness is $\sim 500 \mu \mathrm{m}$ thick, this is $\sim 1-2 \%$ of the thickness, in reasonable agreement with the estimation above.

Shown in Fig. 3B is a graph of the fraction by mass of SiC particles transmitted through the membrane $(\mathrm{T})$, a parameter which is a measure of the membrane's selectivity. For the GF membranes infused with PTFE-NPsonly, we find $\mathrm{T}$ to be very close to the value of 0.06 measured for the GF-only membranes showing the PTFE nanoparticles to have very little impact on the selectivity. Combined with the flow rate data in Fig. $3 \mathrm{~A}$, this implies that although much of the pores are being blocked by PTFE nanoparticles and so reducing the flow rate, some very large pores exist which allow a significant amount of the $\mathrm{SiC}$ nanoparticles to pass through the membrane.

However, the GF membranes filled with a combination of PTFE-NPs/graphene show a completely different picture. The transmitted fraction of $\mathrm{SiC}$ particles falls dramatically with $\Delta \mathrm{M} / \mathrm{M}_{\mathrm{GF}}$, reaching values as low as $\sim 10^{-4}$ for polymer/graphene loadings above $10 \%$. This high degree of selectivity shows that presence of graphene to result in a significant reduction in pore size as $\Delta \mathrm{M} / \mathrm{M}_{\mathrm{GF}}$ increases.

Any practical membrane requires high values of $\mathrm{F}$, coupled with low values of $\mathrm{T}$ (i.e. high flow rate and high selectivity). To investigate this, we plotted T versus F in Fig. 3C for both GF/PTFE-NP membranes as well as GF/ $\mathrm{PTFE} / \mathrm{Graphene}$ membranes. To put this data in context, we measured $\mathrm{F}$ and $\mathrm{T}$ for a range of labscale commercial membranes fabricated from teflon, polypropylene, polyester, nylon and cellulose. We find the GF/PTFE/Graphene membranes to have combinations of $\mathrm{F}$ and $\mathrm{T}$ which are superior to all the commercial membranes tested.

Given that we require high values of $\mathrm{F}$ coupled with low $\mathrm{T}$, we can crudely express the performance of the membrane via F/T. This parameter is plotted in Fig. 3D as a function of membrane density. By this metric, Fig. 3D clearly shows the GF/PTFE/Graphene membranes to be far superior to both GF/PTFE membranes and commercial membranes. Interestingly, F/T shows a clear peak for GF/PTFE/Graphene membranes with densities in the range $200-230 \mathrm{~kg} / \mathrm{m}^{3}$. These densities are just above that of GF-only networks $\left(\sim 200 \mathrm{~kg} / \mathrm{m}^{3}\right)$, implying that the ideal situation involves a very thin surface layer of graphene and PTFE which can block most of the particles without significantly reducing the flow rate.

Size selectivity. It is likely that it is the smallest $\mathrm{SiC}$ nanoparticles which are transported through the membrane with the largest ones becoming trapped. This means that the size of the transmitted particles is likely to be related to the size of the pores within the membrane. In turn, because the PTFE-NP-only membranes appeared to contain large pores, we expect a reduction in pore size as the graphene content is increased. To test this, we filtered a stock $\mathrm{SiC} /$ ethanol dispersion through a range of composite membranes prepared from soak dispersions with various values of $\mathrm{C}_{\mathrm{G}} /\left(\mathrm{C}_{\mathrm{G}}+\mathrm{C}_{\mathrm{P}}\right)$ (and so different $\left.\Delta \mathrm{M} / \mathrm{M}_{\mathrm{GF}}\right)$. In each case, we measured the transmitted fraction and used TEM to measure the diameter distribution of $\mathrm{SiC}$ nanoparticles in the filtrate. The same procedure was carried out in commercial membranes made of PET (nominal pore size $200 \mathrm{~nm}$ ) and alumina (nominal pore size $50 \mathrm{~nm}$ ).

Shown in Fig. $4 \mathrm{~A}$ is the transmitted fraction for a subset of composite membranes plotted versus $\mathrm{C}_{\mathrm{G}} /\left(\mathrm{C}_{\mathrm{G}}+\mathrm{C}_{\mathrm{P}}\right)$ of the soak dispersion (estimated $\Delta \mathrm{M} / \mathrm{M}_{\mathrm{GF}}$ is given in the top axis). This data is consistent with Fig. $3 \mathrm{~B}$ and confirms the selectivity to improve dramatically with mass uptake. To test size selectivity, we first used TEM to measure the diameter distribution of the stock dispersion of $\mathrm{SiC}$ nanoparticles in ethanol, as shown in Fig. 4B (see insets for typical images). The distribution spans between $\sim 25 \mathrm{~nm}$ and $250 \mathrm{~nm}$ with a mean at $112 \mathrm{~nm}$.

When the stock dispersion is filtered through a membrane, only those nanoparticles small enough to pass through the pores get transmitted to the filtrate. Shown in Fig. $4 \mathrm{C}$ is the measured size distribution for SiC nanoparticles filtered through a commercial PET membrane (nominal pore size $200 \mathrm{~nm}$ ). The distribution is quite similar to the stock (mean of $97 \mathrm{~nm}$ ) showing that all nanoparticle sizes can travel through such large pores size networks. We performed the same test with alumina membranes (nominal pore size $50 \mathrm{~nm}$ ). Due to their small pore size, such membranes should only pass very small nanoparticles. However, such high selectivity means these membranes are very expensive ( $€ 13$ per membrane of diameter of $25 \mathrm{~mm}$, www.sigmaaldrich.com). As shown in Fig. 4D, the diameter distribution of the transmitted particles is much narrower than that associated with either the stock or the filtrate from the PET membrane, spanning between $\sim 5$ and $\sim 50 \mathrm{~nm}$ with a mean at $21 \mathrm{~nm}$.

The equivalent diameter distributions for nanoparticles transmitted through GF/PTFE/graphene composite membranes is shown in Fig. $4 \mathrm{E}-\mathrm{G}$ for membranes prepared from soak dispersions with $\mathrm{C}_{\mathrm{G}} /\left(\mathrm{C}_{\mathrm{G}}+\mathrm{C}_{\mathrm{P}}\right)$ values of $5 \%, 9 \%$ and $47 \%$ respectively. It is clear from these graphs that the diameter distributions of the transmitted nanoparticles falls sharply as the graphene content in the soak dispersion increases. To show this quantitatively, 
A

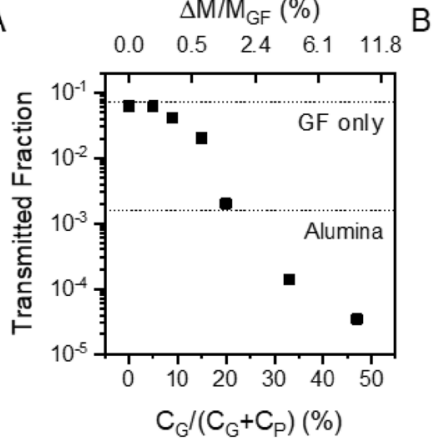

$\mathrm{E}$

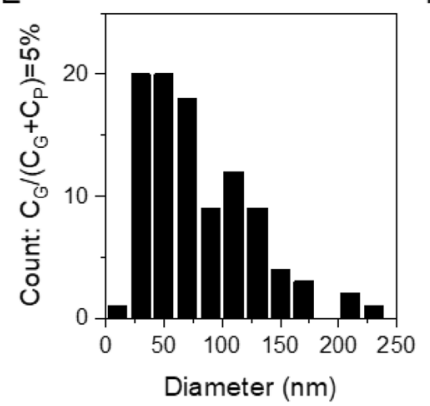

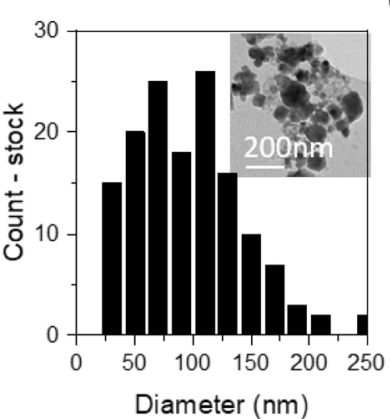

C

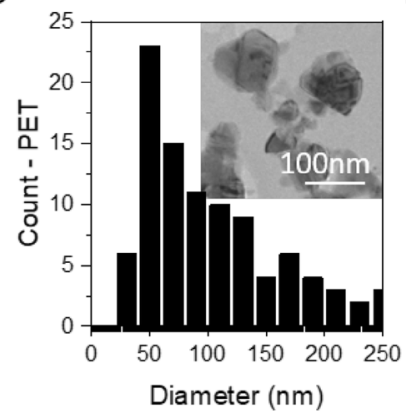

D

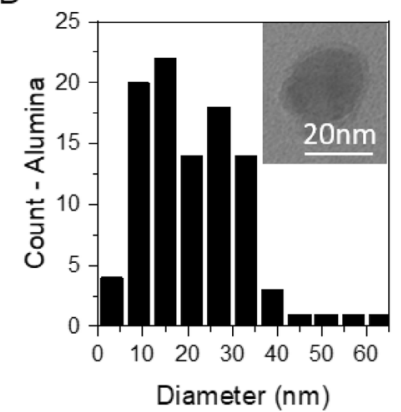

$\mathrm{F}$

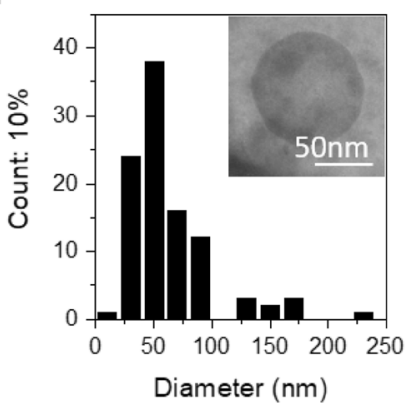

G

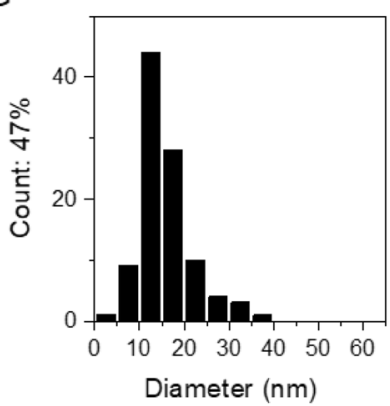

$\mathrm{H}$

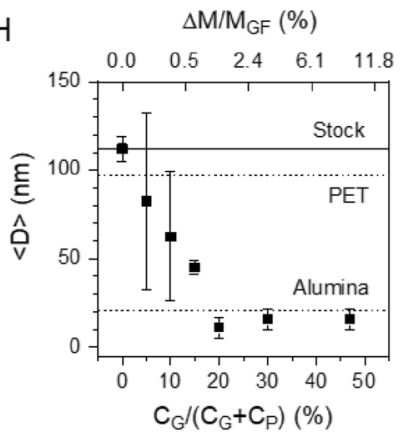

Figure 4. Size of transmitted $\mathrm{SiC}$ particles. (A) Transmitted fraction as a function of $\mathrm{C}_{\mathrm{G}} /\left(\mathrm{C}_{\mathrm{G}}+\mathrm{C}_{\mathrm{P}}\right)$ as an indicator of nominal graphene content. The top axis is a non-linear scale and shows equivalent values of mass uptake, $\Delta \mathrm{M} / \mathrm{M}_{\mathrm{GF}}$ (B) Diameter distributions of $\mathrm{SiC}$ nanoparticles in the stock dispersion $(0.2 \mathrm{mg} / \mathrm{ml})$ used to perform the filtration experiments to probe filter properties. (C-F) Diameter distributions of $\mathrm{SiC}$ particles found in the filtrate (i.e. transmitted through the filter) for stock dispersions filtered through (C) a PET membrane (nominal pore size $100 \mathrm{~nm}$ ), (D) an alumina membrane (nominal pore size $20 \mathrm{~nm}$ ), (E-G) GF membranes filled with PTFE and graphene from soak dispersions with $\mathrm{C}_{\mathrm{G}} /\left(\mathrm{C}_{\mathrm{G}}+\mathrm{C}_{\mathrm{P}}\right)$ values of $(\mathbf{E}) 5 \%,(\mathbf{F}) 9 \%$ and $(\mathbf{G}) 47 \%$. The insets in (B-D,F) show sample TEM images. (H) Mean transmitted $\mathrm{SiC}$ particle diameter plotted versus $\mathrm{C}_{\mathrm{G}} /$ $\left(\mathrm{C}_{\mathrm{G}}+\mathrm{C}_{\mathrm{P}}\right)$ as an indicator of nominal graphene content. The top axis is a non-linear scale and shows equivalent values of mass uptake, $\Delta \mathrm{M} / \mathrm{M}_{\mathrm{GF}}$. The solid horizontal line in $(\mathbf{H})$ represents the mean SiC particle size in the stock. The dashed horizontal lines in $(\mathbf{H})$ represent the mean particle size transmitted through the PET and Alumina membranes. The PET membrane studied represents the polymer membrane with the smallest nominal pore size. In $(\mathbf{A}, \mathbf{H})$ the $0 \%$ data point represents a GF membrane infiltrated with PTFE-NPs only.

we measured the $\mathrm{SiC}$ nanoparticle diameter distributions for filtrates which passed through a range of membranes prepared from soak dispersions with various $\mathrm{C}_{\mathrm{G}} /\left(\mathrm{C}_{\mathrm{G}}+\mathrm{C}_{\mathrm{P}}\right)$. We have plotted the mean transmitted particle diameter, $<\mathrm{D}>$, versus $\mathrm{C}_{\mathrm{G}} /\left(\mathrm{C}_{\mathrm{G}}+\mathrm{C}_{\mathrm{P}}\right)$ in Fig. $4 \mathrm{H}$. For $\mathrm{C}_{\mathrm{G}} /\left(\mathrm{C}_{\mathrm{G}}+\mathrm{C}_{\mathrm{P}}\right)=0$ (equivalent to a GF/PTFE-NP membrane) $<\mathrm{D}>$ is virtually identical to that of the stock indicating no particle size selectivity. However, as $\mathrm{C}_{\mathrm{G}} /$ $\left(C_{G}+C_{P}\right)$ is increases, $<D>$ falls sharply before saturating in the range $7-16 \mathrm{~nm}$ for values of $C_{G} /\left(C_{G}+C_{P}\right)$ above $20 \%$. The diameter distribution for the $47 \%$ membrane (Fig. $4 \mathrm{G}$ ) is significantly narrower than that of the alumina membrane and shows no transmission for particles above $40 \mathrm{~nm}$.

This is a significant result: addition of a small amount of graphene and PTFE to extremely cheap GF membranes yields selectivity which is similar to that of much more expensive alumina membranes. Comparing Fig. $4 \mathrm{~A}, \mathrm{H}$ it is clear that alumina-like selectivity performance can be achieved once $\mathrm{C}_{\mathrm{G}} /\left(\mathrm{C}_{\mathrm{G}}+\mathrm{C}_{\mathrm{P}}\right)>20 \%$ which is equivalent to $\Delta \mathrm{M} / \mathrm{M}_{\mathrm{GF}}>1 \%$. However the significantly higher flow rate under these conditions is a significant advantage. This is an extremely small amount of PTFE/graphene suggesting the conversion of low performance GF membranes to high performance composite membranes to be cost effective.

The fit in Fig. 3A suggests the characteristic pore cross-sectional area to be $A_{\mathrm{p}} \sim 6 \times 10^{-14} \mathrm{~m}^{2}$. As discussed above this implies a pore size of $\sqrt{ } A_{p}=250 \mathrm{~nm}$, a value that looks too large in light of the data in Fig. $4 \mathrm{H}$ which implies pore widths of $\sim 20 \mathrm{~nm}$ in our systems. We can reconcile this discrepancy by noting that nanosheet networks would be expected to have slit-shaped pores as defined by the gap between two adjacent aligned nanosheets. If we assume such pores have typical length, $\mathrm{L}_{\mathrm{p}}$ and width, $\mathrm{W}_{\mathrm{p}}$, we can make the approximation that $\mathrm{A}_{\mathrm{P}} \sim \mathrm{L}_{\mathrm{P}} \mathrm{W}_{\mathrm{P}}$. Then taking $\mathrm{A}_{\mathrm{P}} \sim 6 \times 10^{-14} \mathrm{~m}^{2}$ and assuming $\mathrm{W}_{\mathrm{P}} \sim 40 \mathrm{~nm}$ in line with the data in Fig. $4 \mathrm{G}$ allows us to estimate $\mathrm{L}_{\mathrm{P}} \sim 1.5 \mu \mathrm{m}$, a value which is similar to our mean nanosheet length $(\sim 0.6 \mu \mathrm{m})$. This shows our data to be largely self-consistent.

Field trials. While the lab-based testing described above suggests the GF/PTFE/graphene composite membranes to be promising ultrafiltration membranes, it is important to demonstrate their efficacy under more realistic conditions. One important application of ultrafiltration membranes is the removal of particulate impurities from dirty water to produce potable water. Clearly, for water purification in poor regions of the world, it will be 
A
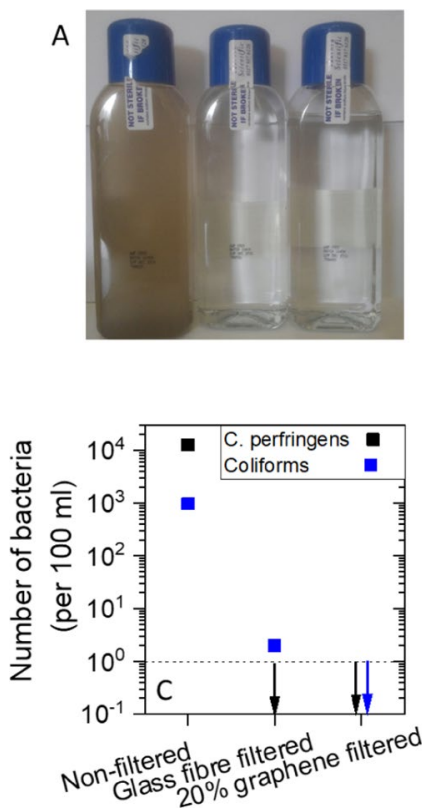
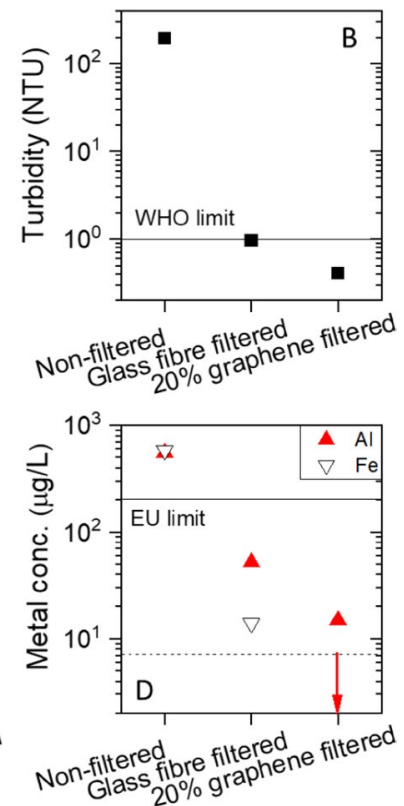

Figure 5. (A) Photograph of water collected from the Grand Canal in Dublin before filtration (left), after filtration through a glass fibre membrane (middle) and after filtration through a $\mathrm{C}_{\mathrm{G}} /\left(\mathrm{C}_{\mathrm{G}}+\mathrm{C}_{\mathrm{P}}\right)=20 \% \mathrm{GF} / \mathrm{PTFE} /$ graphene membrane $\left(\Delta \mathrm{M} / \mathrm{M}_{\mathrm{GF}} \sim 1.3 \%\right.$, right). (B-D) Impurity metrics as measured by a commercial water purity testing firm for the three water samples in (A). In (B), turbidity is a measure of visible light scattering and is commonly used to indicate the presence of impurities in water. The unit used is the Nephelometric Turbidity Unit (NTU). In (C), C. perfringens is a bacterium which is a common cause of food poisoning while coliforms are a bacteria group, which are a commonly used indicator of water quality. The number of C. perfringens is reported in colony forming units (CFU) while the number of coliforms is reported as the most probable number (MPN). The solid lines in (B,D) represent the recommended limits set by the WHO and the EU. In (D) the graphene-based membrane data was below the Fe detection limit of the experiment (dashed line) as indicated by the arrow. In (D), both the GF and graphene-based membrane data were below the C. perfringens detection limit while the graphene-based membrane datum was below the coliforms detection limit as indicated by the arrows (detection limit shown as dashed line).

very important to achieve high selectivity at low cost. Thus, it is important to assess the utility of our composite membranes for water purification.

To test this, we collected dirty water from the Grand Canal, an inner-city canal in Dublin which is not known for its water purity. The collected water was brown and turbid as shown in the left hand container in Fig. 5A. Portions of the dirty water were filtered through a glass fibre membrane (see Fig. 5A middle) and a $\mathrm{C}_{\mathrm{G}} /$ $\left(\mathrm{C}_{\mathrm{G}}+\mathrm{C}_{\mathrm{P}}\right)=20 \% \mathrm{GF} / \mathrm{PTFE} /$ graphene membrane $\left(\Delta \mathrm{M} / \mathrm{M}_{\mathrm{GF}} \sim 1.3 \%\right.$, see Fig. $5 \mathrm{~A}$ right $)$. After filtration, both samples appeared clean and transparent to the naked eye.

To perform quantitative purity data, we sent both dirty and clean samples to a commercial water testing company (City Analysts Limited) for analysis. Of the parameters tested, probably the most interesting are the turbidity, the bacteria content and the metal (Fe and $\mathrm{Al}$ ) content.

The turbidity is a measure of light scattered from a liquid and is often used as a measure of suspended solids content. In the as-collected, un-filtered (Fig. 5B) water the turbidity was 196 NTU (Nephelometric Turbidity Unit), $\times 200$ higher than the WHO recommended limit ${ }^{43}$ for drinking water (1 NTU). Filtration through the untreated GF membrane brought the turbidity down to 0.97 NTU, just below the WHO limit. However, filtration through the composite membrane brought the turbidity down even further, to 0.4 NTU. This indicates that the composite membrane is approximately $\times 2.5$ times more effective at removing solids than the GF membrane.

The concentration of two types of bacteria, C. perfringens and coliforms, are given in Fig. 5C. C. perfringens is a bacterium which is a common cause of food poisoning while coliforms are a bacteria group, which are a commonly used indicator of water quality. In the untreated water, the concentrations of C. perfringens and coliforms respectively are 12,600 and 970 units per $100 \mathrm{ml}$. After filtration through the GF membrane the C. perfringens concentration had fallen below the detection limit (i.e. $<1$ per $100 \mathrm{ml}$ ) while the coliform concentration had fallen to 2 per $100 \mathrm{ml}$. However, after filtration through the composite membrane, both bacteria concentrations had fallen below the detection limit. This shows the composite membrane is at least twice as effective at removing coliform as the GF membrane.

Water quality testing also gives the concentration of metal, usually iron and aluminium. In water, iron is found both as $\mathrm{Fe}^{2+}$ ions and in the form of compounds such as iron oxides ${ }^{44}$. Similarly, aluminium in the form of a range of compounds, most commonly salts ${ }^{45}$. In the untreated water (Fig. 5D), the concentrations of iron and aluminium were very similar at 583 and $550 \mu \mathrm{g} / \mathrm{L}$, significantly above the EU recommended limit for drinking water of $200 \mu \mathrm{g} / \mathrm{L}^{46}$. After filtration through the GF membrane the concentrations fell to 14.5 and $52 \mu \mathrm{g} / \mathrm{L}$ respectively. 
However, after filtration through the composite membrane the iron concentration fell below the detection limit $(7.2 \mu \mathrm{g} / \mathrm{L})$ while the aluminium concentration fell to $15 \mu \mathrm{g} / \mathrm{L}$. This means the composite membrane is at least twice as effective at removing iron and $\times 3.5$ times as effective at removing aluminium as the GF-only membrane.

\section{Conclusion}

In conclusion, we have demonstrated a method to infuse combinations of liquid-exfoliated graphene nanosheets and water-stable Teflon nanoparticles into commercial glass-fibre membranes. Thermal annealing causes the coalescence of the PTFE, resulting in a mechanically stable structure. These composite membranes are considerably stronger and stiffer than neat glass-fibre membranes and mechanically competitive with commercial membranes. Although the addition of graphene/PTFE does reduce the flow rate compared to the neat glass-fibre membranes, it remains superior to many commercial membranes. However, the selectivity, as measured by the fraction of the probe nanoparticles transmitted through the membrane, increased by $\times 10^{3}$ compared to glass-fibres alone. We found the combination of flow rate and selectivity to be far superior to any of the commercial membranes tested under similar conditions. By measuring the diameter distribution of $\mathrm{SiC}$ nano-particles remaining in the permeate which was transmitted through the composite membranes, we estimate the mean pore size to be $\sim 20 \mathrm{~nm}$, similar to commercial alumina membranes which are relatively expensive. In a field trial we used our membranes to purity dirty canal water. After filtration, we found reductions in turbidity and metal content of almost $\times 3000$ and $\times 100$ respectively to well below international limits and a complete removal of bacteria. We believe this technology represents a potentially low-cost route to reasonably high performance ultra-filtration membranes.

\section{Methods}

Graphite (Sigma Aldrich) was ultra-sonicated using ultrasonic processor UP200S Hielscher at a concentration of $120 \mathrm{mg} / \mathrm{ml}$ in TWEEN/water solution $(4 \mathrm{mg} / \mathrm{ml})$ for $72 \mathrm{~h}$. The resultant dispersion was centrifuged at 500 RPM for $45 \mathrm{~min}$ and the supernatant collected. The graphene nanosheets within the supernatant were collected by filtration through a Sterlitech 0.45 micron PVDF membrane. The resultant filter cake was re-dispersed in deionised water by ultrasonic tip sonication for $1 \mathrm{~h}$ at a concentration of $40 \mathrm{mg} / \mathrm{ml}$ to yield a stock dispersion. The methods used here are very similar to those previously reported by use for the exfoliation of graphene ${ }^{47,48}$. In addition, PTFE nanoparticles dispersion was supplied by 3 M Dyneon, (Dispersion TF 5035GZ). The graphene stock dispersion was mixed with the PTFE nanoparticle dispersion in various proportions such that the liquid volume was always $10 \mathrm{ml}$, the PTFE mass was always $100 \mathrm{mg}$ but the graphene mass varied between 0 and $150 \mathrm{mg}$. After mixing, these composite dispersions were sonicated for $15 \mathrm{~min}$ to homogenise in a low power sonic bath. The graphene/PTFE/water dispersions $(10 \mathrm{ml})$ were then poured into Petri dishes and a pre-weighed glass fibre (www.sterlitech.com) membrane placed in each to soak. After 45 min soaking, the membrane was taken out of the petri dish and placed on a tissue paper for $15 \mathrm{~min}$ to remove excess water. The resultant GF/graphene/ PTFE membranes were placed in an oven at $50^{\circ} \mathrm{C}$ overnight to remove any remaining water and then annealed in a furnace at $420^{\circ} \mathrm{C}$ for $45 \mathrm{~min}$ to allow the PTFE particles to coalesce. The graphene/PTFE/GF and control membranes were sonicated in ethanol to remove any unbound graphene or PTFE before being dried in ambient conditions for $45 \mathrm{~min}$. Each membrane was then weighted again to yield the mass of graphene + PTFE deposited on the GF. The process yielded a set of graphene/PTFE/GF membranes with various graphene/PTFE ratios.

The membranes were characterised by filtering $10 \mathrm{ml}$ dispersions $(0.2 \mathrm{mg} / \mathrm{ml})$ of SiC nanoparticles in ethanol under pressure ( $60 \mathrm{kPa}$, controlled via the pump) through the membrane and measuring both the filtration time, to yield the flow rate, and the concentration of $\mathrm{SiC}$ in the filtrate, to yield the transmitted fraction. The $\mathrm{SiC}$ concentration was measured via the optical absorbance of the dispersion at $550 \mathrm{~nm}$ using an absorption coefficient of $13.85 \mathrm{ml} \cdot \mathrm{mg}^{-1} \cdot \mathrm{cm}^{-1}$. The transmitted fraction is defined as the fractional mass of SiC particles which were transported through the membrane. This procedure was repeated three time for each membrane (i.e. each mass fraction) and the flow rate and transmitted fraction data averaged.

The thicknesses of all membranes were measured using digital micrometer with the untreated GF membranes displaying a thickness of $525 \pm 25 \mu \mathrm{m}$. Diameters were recorded using a digital calipers. Mechanical properties were measured using a Zwick Z0.5 ProLine Tensile Tester (100 N Load Cell) tensile tester with a gauge length of $6 \mathrm{~mm}$ and strain rate of $1 \mathrm{~mm} / \mathrm{min}$. TEM grids were prepared by first diluting the dispersions and then dropcasting them onto holey carbon grids ( $\mathrm{Cu} 400 \mathrm{mesh}$ ). Residual solvent was removed from the grids by drying in a vacuum oven. Bright field TEM images were obtained using a Jeol 2100 operating at $200 \mathrm{keV}$. Scanning electron microscopy on the composites was undertaken using a Zeiss Ultra Plus. Using the SE2 detector, samples were examined at a working distance of $\sim 2 \mathrm{~mm}$, with a $30 \mu \mathrm{m}$ aperture and a voltage of $5 \mathrm{kV}$. Samples for cross-sectional imaging were prepared by freeze fracture. Water purity tests were performed by the commercial testing company City Analysts Limited.

Received: 2 April 2020; Accepted: 30 October 2020

Published online: 03 December 2020

\section{References}

1. Drinking water, <https://www.who.int/news-room/fact-sheets/detail/drinking-water> (2019).

2. The united nations world water development report $2015,<$ https://sustainabledevelopment.un.org/content/documents/1711W ater\%20for\%20a\%20Sustainable\%20World.pdf> (2015).

3. Prüss-Üstün, A., Bos, R., Fiona Gore, F. \& Bartram, J. Safer Water, Better Health: Costs, Benefits and Sustainability of Interventions to Protect and Promote Health. (The World Health Organisation, 2008).

4. Forster, J. Water use in industry, <https://ec.europa.eu/eurostat/statistics-explained/index.php/Archive:Water_use_in_industry> (2014). 
5. Hilal, N. \& Wright, C. J. Exploring the current state of play for cost-effective water treatment by membranes. NPJ Clean Water $\mathbf{1}$, 8. https://doi.org/10.1038/s41545-018-0008-8 (2018).

6. Xiong, J., Huo, P. F. \& Ko, F. K. Fabrication of ultrafine fibrous polytetrafluoroethylene porous membranes by electrospinning. J. Mater. Res. 24, 2755-2761. https://doi.org/10.1557/jmr.2009.0347 (2009).

7. Bhardwaj, N. \& Kundu, S. C. Electrospinning: A fascinating fiber fabrication technique. Biotechnol. Adv. 28, 325-347. https://doi. org/10.1016/j.biotechadv.2010.01.004 (2010).

8. Huang, Z. M., Zhang, Y. Z., Kotaki, M. \& Ramakrishna, S. A review on polymer nanofibers by electrospinning and their applications in nanocomposites. Compos. Sci. Technol. 63, 2223-2253. https://doi.org/10.1016/s0266-3538(03)00178-7 (2003).

9. Gao, W. et al. Membrane fouling control in ultrafiltration technology for drinking water production: A review. Desalination 272, 1-8. https://doi.org/10.1016/j.desal.2011.01.051 (2011).

10. Zhang, R. N. et al. Antifouling membranes for sustainable water purification: Strategies and mechanisms. Chem. Soc. Rev. 45, 5888-5924. https://doi.org/10.1039/c5cs00579e (2016).

11. Mulder, M. Basic Principles of Membrane Technology 2nd edn. (Kluwer Academic, Dordrecht, 1996).

12. Anderson, M. A., Gieselmann, M. J. \& Xu, Q. Y. Titania and alumina ceramic membranes. J. Membr. Sci. 39, 243-258. https://doi. org/10.1016/s0376-7388(00)80932-1 (1988).

13. Van Gestel, T. et al. Alumina and titania multilayer membranes for nanofiltration: Preparation, characterization and chemical stability. J. Membr. Sci. 207, 73-89. https://doi.org/10.1016/s0376-7388(02)00053-4 (2002).

14. Vereb, G. et al. Highly efficient bacteria inactivation and phenol degradation by visible light irradiated iodine doped TiO2. Appl. Catal. B-Environ. 129, 194-201. https://doi.org/10.1016/j.apcatb.2012.08.037 (2013).

15. Rashid, M. H. O. \& Ralph, S. F. Carbon nanotube membranes: Synthesis, properties, and future filtration applications. Nanomaterials 7, 28. https://doi.org/10.3390/nano7050099 (2017).

16. Xu, C., Cui, A. J., Xu, Y. L. \& Fu, X. Z. Graphene oxide-TiO2 composite filtration membranes and their potential application for water purification. Carbon 62, 465-471. https://doi.org/10.1016/j.carbon.2013.06.035 (2013).

17. Homaeigohar, S. \& Elbahri, M. Graphene membranes for water desalination. NPG Asia Mater. 9, 16. https://doi.org/10.1038/ am.2017.135 (2017).

18. Thebo, K. H. et al. Highly stable graphene-oxide-based membranes with superior permeability. Nat. Commun. 9, 8. https://doi. org/10.1038/s41467-018-03919-0 (2018).

19. Dikin, D. A. et al. Preparation and characterization of graphene oxide paper. Nature 448, 457-460. https://doi.org/10.1038/natur e06016 (2007).

20. Cheng, M. M. et al. Recent developments in graphene-based/nanometal composite filter membranes. RSC Adv. 7, 47886-47897. https://doi.org/10.1039/c7ra08098k (2017).

21. Zhou, K. G. et al. Electrically controlled water permeation through graphene oxide membranes. Nature 559, 236. https://doi. org/10.1038/s41586-018-0292-y (2018).

22. Joshi, R. K., Alwarappan, S., Yoshimura, M., Sahajwalla, V. \& Nishina, Y. Graphene oxide: The new membrane material. Appl. Mater. Today 1, 1-12. https://doi.org/10.1016/j.apmt.2015.06.002 (2015).

23. Huang, T. F., Puspasari, T., Nunes, S. P. \& Peinemann, K. V. Ultrathin 2D-layered cyclodextrin membranes for high-performance organic solvent nanofiltration. Adv. Funct. Mater. https://doi.org/10.1002/adfm.201906797 (2019).

24. Nair, R. R., Wu, H. A., Jayaram, P. N., Grigorieva, I. V. \& Geim, A. K. Unimpeded permeation of water through helium-leak-tight graphene-based membranes. Science 335, 442-444. https://doi.org/10.1126/science.1211694 (2012).

25. Sun, P. Z. et al. Selective ion penetration of graphene oxide membranes. ACS Nano 7, 428-437. https://doi.org/10.1021/nn304471w (2013).

26. Hegab, H. M. \& Zou, L. D. Graphene oxide-assisted membranes: Fabrication and potential applications in desalination and water purification. J. Membr. Sci. 484, 95-106. https://doi.org/10.1016/j.memsci.2015.03.011 (2015).

27. Xu, Z. W. et al. Organosilane-functionalized graphene oxide for enhanced antifouling and mechanical properties of polyvinylidene fluoride ultrafiltration membranes. J. Membr. Sci. 458, 1-13. https://doi.org/10.1016/j.memsci.2014.01.050 (2014).

28. Yu, L. et al. Preparation and characterization of HPEI-GO/PES ultrafiltration membrane with antifouling and antibacterial properties. J. Membr. Sci. 447, 452-462. https://doi.org/10.1016/j.memsci.2013.07.042 (2013).

29. Alhusein, N., Scott, J., Kasprzyk-Hordern, B. \& Bolhuis, A. Development of a filter to prevent infections with spore-forming bacteria in injecting drug users. Harm Reduct. J. 13, 8. https://doi.org/10.1186/s12954-016-0122-1 (2016).

30. Padilla, C. C. et al. Standard filtration practices may significantly distort planktonic microbial diversity estimates. Front. Microbiol. 6, 10. https://doi.org/10.3389/fmicb.2015.00547 (2015).

31. Bonaccorso, F., Bartolotta, A., Coleman, J. N. \& Backes, C. 2D-crystal-based functional inks. Adv. Mater. 28, 6136-6166. https:// doi.org/10.1002/adma.201506410 (2016).

32. Niu, L. Y. et al. Production of two-dimensional nanomaterials via liquid-based direct exfoliation. Small 12, 272-293. https://doi. org/10.1002/smll.201502207 (2016).

33. Dullien, F. A. L., Press, A., Science, E. \& Technology,. Porous Media: Fluid Transport and Pore Structure (Academic Press, Cambridge, 1979).

34. Wang, Z., Liang, Z. Y., Wang, B., Zhang, C. \& Kramer, L. Processing and property investigation of single-walled carbon nanotube (SWNT) buckypaper/epoxy resin matrix nanocomposites. Composit. Part A-Appl. Sci. Manuf. 35, 1225-1232. https://doi. org/10.1016/j.compositesa.2003.09.029 (2004).

35. Gou, J. H. Single-walled nanotube bucky paper and nanocomposite. Polym. Int. 55, 1283-1288. https://doi.org/10.1002/pi.2079 (2006).

36. Coleman, J. N. et al. Improving the mechanical properties of single-walled carbon nanotube sheets by intercalation of polymeric adhesives. Appl. Phys. Lett. 82, 1682-1684. https://doi.org/10.1063/1.1559421 (2003).

37. Kinloch, I. A., Suhr, J., Lou, J., Young, R. J. \& Ajayan, P. M. Composites with carbon nanotubes and graphene: An outlook. Science 362, 547-553. https://doi.org/10.1126/science.aat7439 (2018).

38. Qin, Z., Jung, G. S., Kang, M. J. \& Buehler, M. J. The mechanics and design of a lightweight three-dimensional graphene assembly. Sci. Adv. https://doi.org/10.1126/sciadv.1601536 (2017).

39. Ni, N. et al. Understanding mechanical response of elastomeric graphene networks. Sci. Rep. 5, 13712. https://doi.org/10.1038/ srep13712 (2015).

40. Mecklenburg, M. et al. Aerographite: Ultra lightweight, flexible nanowall, carbon microtube material with outstanding mechanical performance. Adv. Mater. 24, 3486-3490. https://doi.org/10.1002/adma.201200491 (2012).

41. Qiu, L., Liu, J. Z., Chang, S. L. Y., Wu, Y. \& Li, D. Biomimetic superelastic graphene-based cellular monoliths. Nat. Commun. 3, 1241. https://doi.org/10.1038/ncomms2251 (2012).

42. Costa, A. Permeability-porosity relationship: A reexamination of the Kozeny-Carman equation based on a fractal pore-space geometry assumption. Geophys. Res. Lett. https://doi.org/10.1029/2005gl025134 (2006).

43. Turbidity, <http://www.lenntech.com/turbidity.htm\#ixzz3R3yPreK7> (

44. Iron in drinking water, <https://www.who.int/water_sanitation_health/dwq/chemicals/iron.pdf> (1996).

45. Aluminium in Drinking-water, <http://apps.who.int/iris/bitstream/handle/10665/75362/WHO_SDE_WSH_03.04_53_eng. pdf? sequence $=1$ \&ua $=1>(1998)$.

46. European union (drinking water) regulation 2014, <http://www.irishstatutebook.ie/eli/2014/si/122/made/en/pdf> (2014). 
47. Khan, U. et al. Size selection of dispersed, exfoliated graphene flakes by controlled centrifugation. Carbon 50, 470-475. https:// doi.org/10.1016/j.carbon.2011.09.001 (2012).

48. Khan, U. et al. Solvent-exfoliated graphene at extremely high concentration. Langmuir 27, 9077-9082. https://doi.org/10.1021/ la201797h (2011).

\section{Acknowledgements}

We acknowledge financial support from the SFI-funded AMBER research centre (SFI/12/RC/2278) and from the European Union Seventh Framework Programme under grant agreements n 785219 Graphene Flagship-Core 2. C Boland is grateful for financial support through CRANN.

\section{Author contributions}

U.K., S.B., C.S.B. contributed to acquisition, analysis, or interpretation of data. U.K. and J.N.C. conceived and designed the work. J.N.C. analysed data and wrote the paper.

\section{Competing interests}

The authors declare no competing interests.

\section{Additional information}

Correspondence and requests for materials should be addressed to J.N.C.

Reprints and permissions information is available at www.nature.com/reprints.

Publisher's note Springer Nature remains neutral with regard to jurisdictional claims in published maps and institutional affiliations.

(c) (1) Open Access This article is licensed under a Creative Commons Attribution 4.0 International License, which permits use, sharing, adaptation, distribution and reproduction in any medium or format, as long as you give appropriate credit to the original author(s) and the source, provide a link to the Creative Commons licence, and indicate if changes were made. The images or other third party material in this article are included in the article's Creative Commons licence, unless indicated otherwise in a credit line to the material. If material is not included in the article's Creative Commons licence and your intended use is not permitted by statutory regulation or exceeds the permitted use, you will need to obtain permission directly from the copyright holder. To view a copy of this licence, visit http://creativecommons.org/licenses/by/4.0/.

(C) The Author(s) 2020 